\title{
¿Hay verdadera innovación en los lanzamientos de nuevos productos? Factores relevantes de éxito y fracaso en el caso colombiano
}

\author{
MARTHA LUCIA CRUZ RINCÓN, MBA ${ }^{1,2}$ \\ Profesora tiempo completo, Universidad Icesi, Colombia. \\ mlcruz@icesi.edu.co \\ RAQUEL PUENTE CASTRO, Ph.D. ${ }^{3}$ \\ Profesora tiempo completo, Universidad Icesi, Colombia. \\ raquel.puente@correo.icesi.edu.co
}

\section{RESUMEN}

El lanzamiento de nuevos productos es determinante para el crecimiento y supervivencia de las organizaciones; sin embargo, su tasa de fracaso es muy alta. El objetivo de la presente investigación exploratoria es evaluar si los ejecutivos en Colombia aplican las mejores prácticas para el lanzamiento de nuevos productos, sus dificultades en implementación y el rol de la innovación en este proceso. A través de entrevistas a altos ejecutivos de mercadeo de doce organizaciones, se encontraron graves deficiencias en el conocimiento de las mejores prácticas. Asimismo, se encontró que la innovación se centra en mejoras incrementales, sin una conciencia del proceso de aprendizaje que conlleve un cambio en la cultura organizacional y la participación de todas las áreas de la compañía.

Palabras clave. Innovación; lanzamiento nuevos productos; fracaso de nuevos productos; mejores prácticas lanzamiento de nuevos productos.

Recibido: 30-jun-11, corregido: 30-abr-12 y aceptado: 26-jun-12

Clasificación JEL: M10; M30

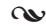

\footnotetext{
1 Este documento fue seleccionado en la convocatoria para enviar artículos, Call for Papers, realizada en el marco del "II Simposio Iberoamericano de Estudios Gerenciales: Una mirada interdisciplinar a la innovación”, organizado por la revista académica Estudios Gerenciales bajo la dirección de la Facultad de Ciencias Administrativas y Económicas de la Universidad Icesi; el evento tuvo lugar los días 12, 13 y 14 de octubre de 2011, en la ciudad de Cali (Colombia). Este documento fue presentado en las sesiones simultáneas del área de "Innovación en marketing".

2 Especial agradecimiento a los estudiantes de la Universidad Icesi que participaron en el proceso, K. Pfizenmaier, N. Lombana, M Ramirez, N. Sánchez y M. Delgado.

3 Autor para correspondencia. Dirigir a: Universidad Icesi, Calle 18 No. 122-135, Pance, Cali, Colombia.
} 


\section{Is there true innovation in the launching of new products? Relevant factors of success and failure in Colombia}

The launching of new products is one of the determining factors of growth and survival of an organization, but the rate of failure is very high. The purpose of this exploratory research work is not only to assess whether executives in Colombia use the best practices for launching new products, but also to review difficulties with implementation and the role of innovation in this process. The findings of interviews conducted among high-level marketing executives at twelve companies reveal that there are serious deficiencies in the knowledge of best practices. They also show that innovation focuses on incremental improvements, but there is a lack of awareness that the learning process can lead to a change in organizational culture and in the participation of all departments in a company.

Keywords. innovation; new product launching; new product failure; best practices for launching new products.

\section{en}

\section{Existe uma verdadeira inovação nos lançamentos de novos produtos? Fatores relevantes de sucesso e fracasso no caso colombiano}

O lançamento de novos produtos é essencial para o crescimento e sobrevivência dos organismos, mas a sua taxa de falha é muito elevada. O objetivo deste estudo exploratório é avaliar se os executivos da Colômbia aplicam as melhores práticas para o lançamento de novos produtos, as dificuldades na implementação e o papel da inovação no processo. Através de entrevistas com executivos de marketing sênior de 12 organizações, se encontraram sérias deficiências no conhecimento das melhores práticas. Assim, descobrimos também que a inovação se concentra em melhorias incrementais, sem uma consciência do processo de aprendizagem que envolve uma mudança na cultura organizacional e participação de todas as áreas da empresa.

Palavras-chave. Inovação; lançamento de produtos novos; insuficiência de novos produtos; melhores práticas de lançamento de novos produtos. 


\section{Introducción}

Existe amplia literatura desarrollada sobre evidencia empírica de cuáles son las mejores prácticas en cuanto a procesos y metodologías para el desarrollo y lanzamiento de nuevos productos. Sin embargo, de cada diez lanzamientos, ocho fracasan (Kuczmarski, 1997). Urban \& Hauser (1980) encontraron que el promedio de probabilidad de éxito en un producto de consumo es sólo del $19 \%$. De lo anterior surge la pregunta, ¿Por qué hay tantos fracasos? Para Ries \& Trout (1989), el 90\% de los productos nuevos fracasan porque su lanzamiento está enfocado a llenar un vacío de la empresa más que un vacío del mercado. Según Clancy \& Shulman (1994), tres de cada diez productos fracasan por debilidad en posicionamiento. Taylor (1983) y Kuczmarski (1997) coinciden en que las principales causas de fracaso pueden estar asociadas a un análisis inadecuado del mercado y la incapacidad de comprender las verdaderas necesidades del consumidor.

$\mathrm{Al}$ parecer el fracaso es parte del aprendizaje necesario en el desarrollo de nuevos productos, pero ¿podrían reducirse las tasas de fracaso si los gerentes encargados de estos desarrollos siguieran una metodología sistemática y lógica que permita revisar, analizar y evaluar cada una de los factores y variables, en el ámbito comercial, técnico y financiero?

En el presente estudio, aunque exploratorio pues se trata de una investigación cualitativa, se evaluará si los ejecutivos a cargo del desarrollo de nuevos productos utilizan las metodologías y las mejores prácticas desarrolladas en el tema, contrastando el marco teórico con las experiencias y percepciones de los ejecutivos entrevistados. Específicamente, se espera obtener hallazgos acerca de cuáles son las dificultades que encaran los ejecutivos para implementar las mejores prácticas en desarrollo de nuevos productos y elaborar recomendaciones que les ayuden a superar los obstáculos que enfrentan en el proceso. Finalmente, se analizará el rol de la innovación en el lanzamiento de nuevos productos, el grado de innovación utilizado, y la percepción de los ejecutivos entrevistados con respecto a la importancia de la misma en la tasa de éxito de los nuevos lanzamientos.

Este documento se divide en cuatro secciones. En la primera sección se presenta el marco teórico que dio pie a la investigación, en la segunda sección se describe la metodología, en la tercera sección los resultados y finalmente se presentan las conclusiones, limitaciones y recomendaciones más importantes que surgen de esta investigación.

\section{Marco teórico}

1.1 Modelos de desarrollo de nuevos productos: factores de éxito y fracaso

¿Qué hace que algunos lanzamientos sean exitosos y otros fracasen? Hay amplia literatura desarrollada buscando responder esta pregunta y, según la experiencia de Clancy \& Shulman (1994), existen cinco razones por las que fracasan los nuevos productos: una estrategia débil y mal articulada de selección de mercado objetivo y de posicionamiento; insatisfacción con el producto, es decir, la incapacidad para cumplir y exceder las expectativas de los consumidores y la oferta de la competencia; un nivel insuficiente de conciencia en el nuevo producto; esfuerzo inadecuado de ventas; $y$ debilidades en el canal de distribución.

Por su parte, Cooper (2007), uno de los autores más prolífero en el tema de desarrollo de nuevos productos, reporta un estudio identificando cuatro variables discriminantes entre los productos ganadores y los perdedores (las primeras cuatro), aunque extiende estos factores a nueve en total: 1. Un proceso de desarrollo de nuevos productos de alta calidad, 2. Estrategia clara 
de la unidad de negocios de desarrollo de nuevos productos, 3. Recursos económicos y humanos adecuados, 4. Inversión en I\&D de nuevos productos, 5. Equipos de proyectos de desarrollo de nuevos productos de alta calidad, 6. Gerencia sénior comprometida e involucrada en el desarrollo de nuevos productos, 7. Cultura y clima de innovación, 8. Equipos de proyectos interfuncionales y 9. Medición y responsabilidad del proyecto a cargo de la gerencia sénior.

A su vez, Brody (1999) integra numerosos estudios y experiencias de expertos en diversas industrias y desarrolla trece requerimientos para tener éxito en el desarrollo de nuevos productos con la finalidad de que sirvan de guía para las empresas (ver Tabla 1).

Definitivamente estas mejores prácticas tienen en común que las empresas que adoptan un proceso formal de desarrollo de nuevos productos, obtienen un mejor desempeño que aquellas que no lo hacen (Booz, Allen \& Hamilton, 1982), lo que incluye tiempo, recursos, investigación, estrategia clara, trabajo coordinado, detalle en la implementación, sistematización de procesos, costos previstos, apoyo de la gerencia y medición de resultados. En este sentido, de la bibliografía sobre desarrollo de nuevos productos, se tomaron dos modelos de desarrollo de nuevos productos que sirvieron de base para la guía y el análisis de la información. El primero de ellos es el presentado por Schnarch (2009) que plantea un esquema de ocho pasos: 1. Objetivos, 2. Búsqueda de idea, 3. Selección de ideas, 4. Desarrollo y prueba de concepto, 5. Análisis de factibilidad de negocio (variables técnicas, económicas y de mercado), 6. Estrategias de mercadeo, 7. Desarrollo de producto, y 8. Lanzamiento del producto y evaluación (ver Gráfico 1).

Tabla 1. Requerimientos de éxito para nuevos productos

(1) Ambiente organizacional apropiado y el soporte de la gerencia alta

(2) Uso de procesos disciplinados de desarrollo de nuevos productos

(3) Equipos de desarrollo de nuevos productos dedicados y con disposición y habilidades para hacer alianzas y outsourcing

(4) Actividades de desarrollo de nuevos productos que comienzan y fluyen a través de las unidades de negocio (UEN)

(5) Comprensión del entorno e identificación de oportunidades que engranan con las competencias del negocio

(6) Identificación de los drivers del consumidor y sus necesidades

(7) Procesos y técnicas para mantener un banco amplio de ideas de desarrollo de nuevos productos

(8) Definición y enfoque claro del producto antes de comenzar el desarrollo de las actividades

(9) Un producto y empaque superior y diferenciado

(10) Investigación de mercados para evaluar el producto y todos los elementos del programa durante el desarrollo del proceso de desarrollo de nuevos productos

(11) Gerencia de la categoría para alinear manufactura y detallistas con foco en el consumidor

(12) Un lanzamiento bien ejecutado

(13) Habilidad de adaptarse, crecer y mejorar para hacer frente a las condiciones del mercado y la competencia

Fuente: De Developing New Food Products for a Changing Marketplace (p. 70), por A. Brody, 1999, Duluth, MI: CRC Press. 
Gráfico 1. Esquema del desarrollo de un nuevo producto

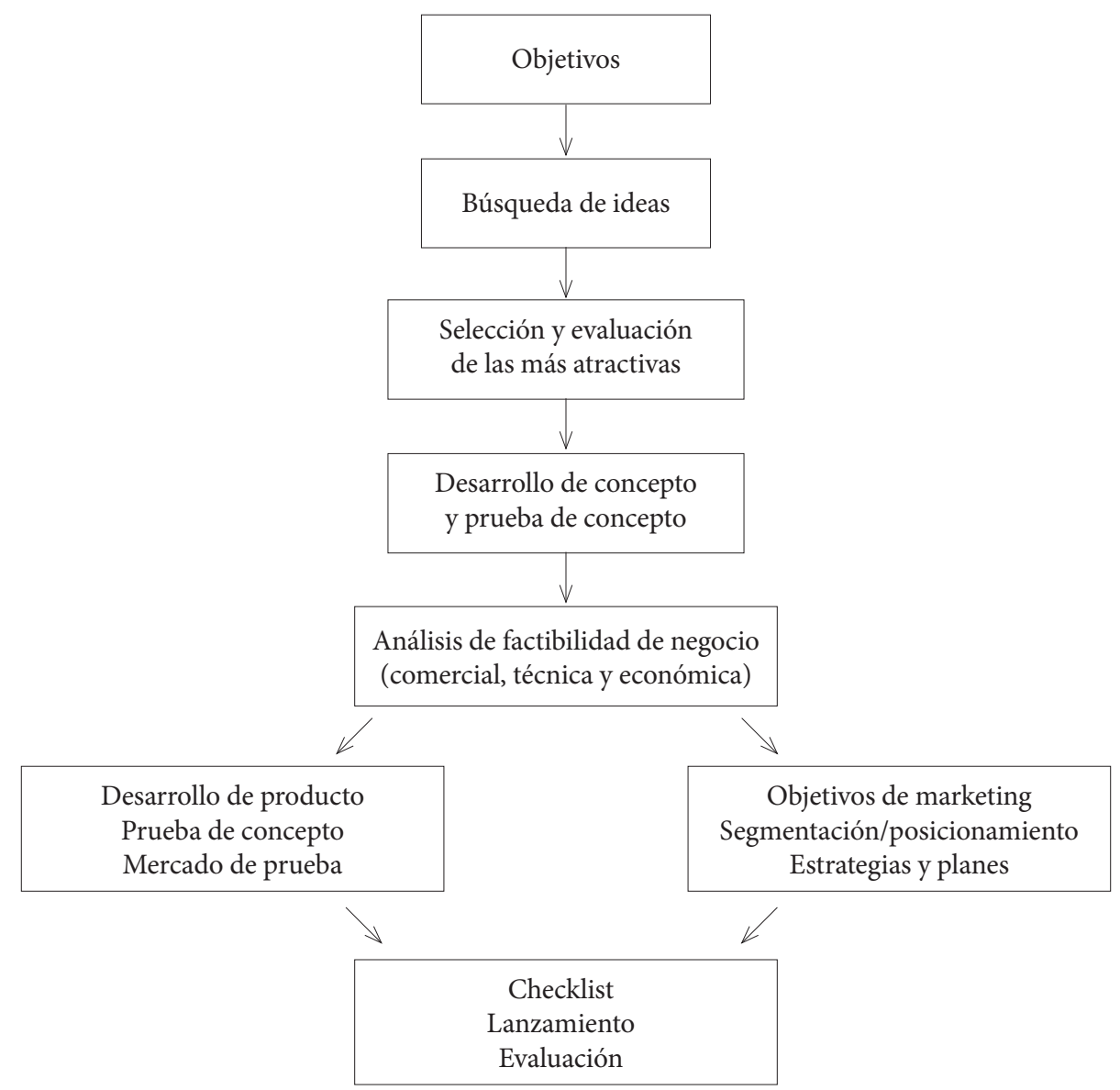

Fuente: De Desarrollo de nuevos productos y empresas (p. 93), por A. Schnarch, 2009, Bogotá: McGraw-Hill.

Schnarch (2009) explica que el proceso debe comenzar con una clara determinación de objetivos, seguido de una búsqueda de ideas en el campo definido, la selección de las más atractivas y pertinentes y finalmente, una evaluación profunda de éstas con el consumidor (prueba de concepto) en la cual se identifican las reacciones, asociaciones e intención de compra que estas generan en el grupo objetivo. Una vez la idea del nuevo producto logra una buena evaluación con el consumidor, se procede a un análisis de factibilidad de negocio (variables técnicas, económicas y de mercado), de la cual se deriva una senda de estrategias de mercadeo y otra de producto, que deben retroalimentarse, ser simultáneas y estar listas al momento del lanzamiento. La metodología debe manejarse de forma flexible, de manera que en cualquier momento se pueda volver a una etapa anterior para cambiar, revisar, modificar, mejorar, postergar o abandonar el proyecto. 
El otro modelo relevante en materia de nuevos productos es el de Cooper \& Kleinschmidt (1993) que propone el método de Stage-Gate. Este método reconoce que el desarrollo de nuevos productos es un proceso que contempla entre cuatro a siete etapas dependiendo de la compañía o división, siendo cinco etapas las más típicas: 1. Determinación del ámbito del proyecto, 2. Construcción del caso de negocios, 3. Desarrollo del producto/servicio, 4. Prueba y validación, y 5. Lanzamiento (ver Gráfico 2). El enfoque de Stage-Gate rompe el proceso de desarrollo de nuevos productos en cinco etapas que son precedidas por una puerta de entrada o punto de decisión. Las puertas cumplen una función de control de calidad para que nada sea dejado al azar.

Buscando lograr una verdadera innovación que integre todos los aspectos del negocio, del mercado y del consumidor, el Stage-Gate parte de una evaluación inicial de la idea del producto evolucionando a un análisis más detallado y profundo, en el que se consideran aspectos fundamentales de mercado, técnicos, financieros y factores claves relativos al negocio. Una vez el proyecto cumple con todos las condiciones definidas, se abre la puerta 3 , permitiendo que la idea evolucione a la etapa de desarrollo técnico e investigación de mercados con el consumidor potencial. Las etapas 4 y 5 identifican la respuesta del consumidor y establecen una estrategia de comercialización coherente para el lanzamiento. Posterior al lanzamiento, el método sugiere un seguimiento y evaluación post-lanzamiento para obtener retroalimentación de parte de los consumidores, canales o intermediarios, reacción de la competencia para la revisión y/o ajuste de

Gráfico 2. Esquema del Modelo Stage-Gate

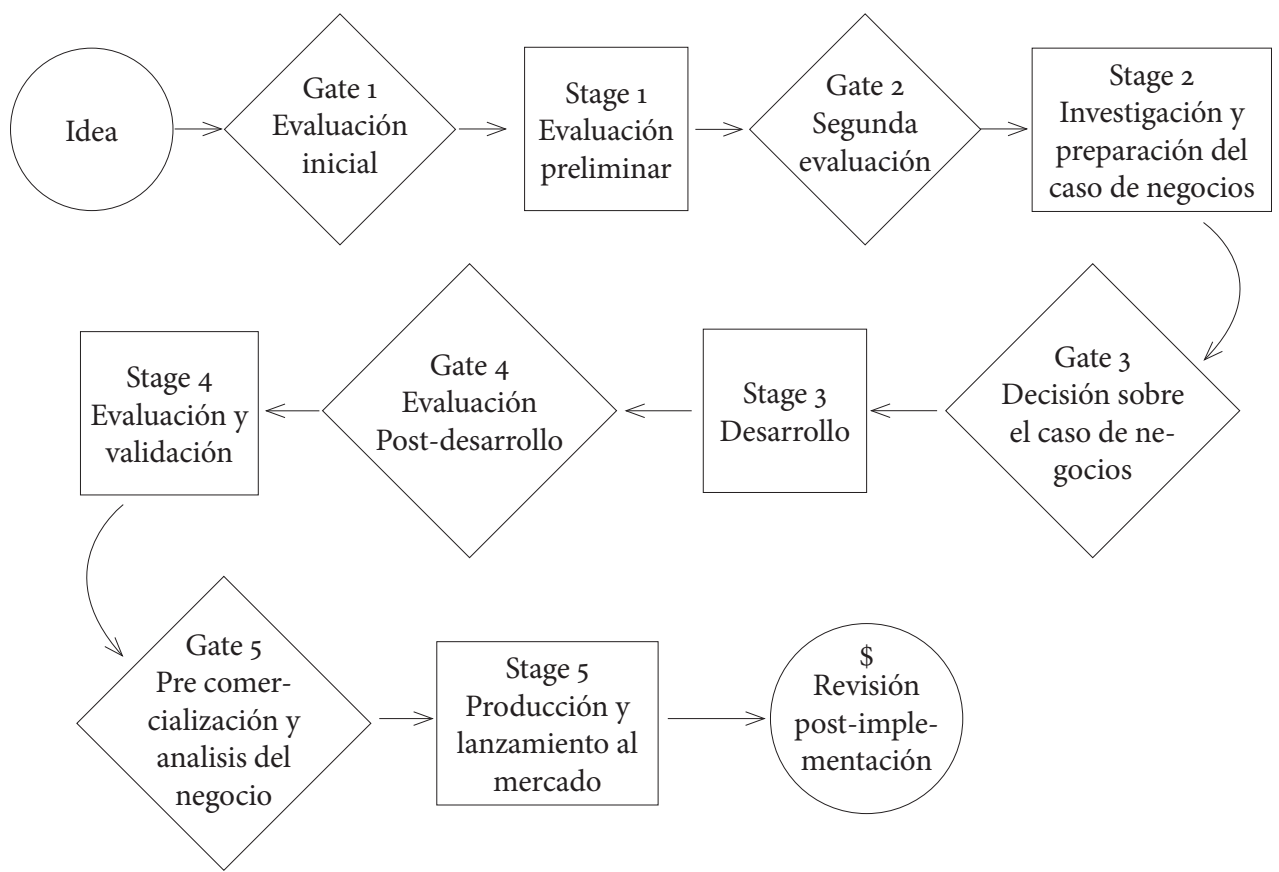

Fuente: De "Stage-Gate Systems: A New Tool for Managing new Products", de R. Cooper, 1990, Business Horizons, 33(3), p. 10. 
las estrategias de mercadeo, como también las proyecciones de acuerdo a la demanda real del nuevo producto.

Cooper (2008) explica que, aunque el modelo parece sencillo, muchos ejecutivos terminan cometiendo errores y haciendo cosas que no deberían y enumera ocho percepciones que el Stage-Gate no debería de ser: 1 . No es un proceso de revisión de fases funcionales, 2. No es un proceso rígido de pasos (es un mapa), 3. No es un mecanismo de control de proyectos, 4. No es data, ni una herramienta estática, 5 . No es un sistema burocrático, 6 . No es un sistema de introducción de data, 7 . No es un proceso de entrega de productos, las primeras tres fases son las más importantes antes del desarrollo del producto, y 8 . No es gerencia de proyectos.

Asimismo, Cooper (2008) recoge los problemas más comunes encarados por los ejecutivos en las organizaciones donde se ha implementado: 1. Manejo y cumplimiento adecuado de los criterios de las compuertas (gates), 2. Aplicación errónea de modelos de reducción de costos a proyectos de innovación, 3. Gerenciar el portafolio de productos sin un proceso de Stage-Gate, 4. Burocracia en el proceso de lanzamiento de la idea, 5. Exceso de importancia a los software como generadores de soluciones, 6 . Sobre-dimensionamiento del alcance de los procesos, y 7 . No hay ganancia sin esfuerzo.

Por todo lo anterior, Cooper $(2006,2008)$ desarrolla un sistema Stage-Gate reforzado, que incluye tomar en cuenta la mayoría de estos errores y malinterpretaciones a la hora de implementarlo y que denomina NexGen Stage-Gate ${ }^{\circledR}$ System (NGS). El NGS incorpora siete principios relacionados con eliminar lo innecesario (lean), rapidez y rentabilidad en el proceso de desarrollo de nuevos productos. A continuación los siete principios explicados brevemente:
- Aproximaciones holísticas. Ocupa el primer lugar en importancia entre los siete principios. Se trata de entender que innovación es un proceso de negocios y trabajo en equipo y no una actividad de investigación y desarrollo.

- Proceso sin desperdicios, escalable y adaptable. Ocupa el segundo lugar en importancia entre los siete principios. Consiste en eliminar todo aquello que no agregue valor (burocracias, desperdicio de tiempo y actividades), eliminando la rigidez y potenciando los procesos para adaptarse a las circunstancias. Cooper $(2006,2008)$ explica que el sistema Stage-Gate no puede ser aplicado como una versión única, sino que para adaptarse el riesgo, el tamaño y la tecnología involucrada, se pueden hablar de tres versiones del modelo: XPress, para proyectos de riesgos moderados; Stage-Gate Lite, para proyectos pequeños con requerimientos simples; y Stage-Gate TD, para proyectos de desarrollo tecnológico.

- Foco en el consumidor. Ocupa el tercer lugar en importancia de los siete principios que diferencian a los negocios más productivos, según la American Productivity \& Quality Center (Edgett, 2007). Consiste en desarrollar nuevos productos para el consumidor que sean diferenciados, que resuelvan la mayoría de sus problemas y que ofrezcan proposiciones de valor superior.

- Arduo trabajo antes de comenzar el desarrollo del producto. Cooper (1990) lo explicaba como "las actividades más importantes, esas que hacen la diferencia entre el éxito y el fracaso, son las primeras actividades en el proceso" (p. 48).

- Desarrollos en espiral. Consiste en crear una primera versión del producto (puede ser virtual) que se apruebe permanentemente con el consumidor, buscando retroalimentación a lo largo de todo el proceso para producir una versión más completa. Este 
bucle se mantiene a lo largo de todo el proceso de Stage-Gate.

- Métricas, responsabilidades y mejoras continuas. Se trata de desarrollar métricas que permitan gerenciar la ejecución de proyectos individuales, incluso luego del lanzamiento y la revisión de los criterios de las compuertas. Cuando se identifican brechas, se realizan sesiones de resolución de problemas, identificando causas y desarrollando acciones correctivas, de esta forma cada nuevo proyecto es ejecutado mejor que el anterior.

- Foco en un portafolio efectivo. No desperdiciar recursos en numerosas iniciativas, comenzar con conceptos sólidos de nuevos productos y sucesivamente remover aquellos débiles aplicando los mecanismos de filtros de las compuertas del sistema. Cooper (2009) dice que las empresas deben concentrarse en desarrollar estrategias de innovación a través de una etapa robusta de descubrimiento y generación de ideas.

Pareciera que estos modelos de desarrollo de nuevos productos se enfocan más en el proceso y la sistematización de cada paso con criterios claros y medibles para avanzar. Lo anterior con el objetivo de reducir los fracasos que, en el nivel de la innovación, presente el proceso y la importancia de la misma como factor de éxito. En este sentido se revisará la literatura sobre innovación, los ámbitos de la misma, sus implicaciones en la consecución de los objetivos de la empresa y la importancia de la misma en el proceso de desarrollo de nuevos productos.

\subsection{La innovación en el desarrollo de nuevos productos}

De acuerdo a Schumpeter (1978) la innovación es la introducción de nuevos bienes, nuevos métodos de producción, la apertura de nuevos mercados, la conquista de nuevas fuentes de producción y el desarrollo de una nueva organización. Innovación tiene que ver con creatividad y nuevas ideas, "el grado de creatividad en el desarrollo de nuevos productos y diseño de procesos" (Sethi, Smith \& Park, 2001, p. 10), muchas veces malinterpretada en las empresas con individuos en oficinas cerradas, que desarrollan nuevos conceptos sujetos a selección, desarrollo y prueba de mercado, y que pueden terminar en un lanzamiento exitoso de productos (Crawford \& Di Benedetto, 2003).

La innovación convencional comienza con productos existentes a los que se les hacen cambios o mejoras, suprimiendo, multiplicando y dividiendo componentes o atributos, con foco en el producto y en la categoría (Goldenberg, Horowitz, Levav \& Mazursky, 2003), logrando introducir innovaciones incrementales que satisfagan las necesidades de los mercados objetivos. Adoptar esta filosofía es asumir que los mercados son océanos en calma con leves y predecibles cambios en los segmentos. Sin embargo, servir nuevos mercados requiere de innovación radical en el diseño de productos y en los modelos de negocios (Chandy \& Tellis, 2000). Es así como aún muchas empresas desarrollan sus estrategias en los océanos rojos - mercados conocidos y estructurados con reglas de competencia establecidas- mientras otras exploran los océanos azules -espacios donde no hay competencia y donde es posible crear demanda y satisfacer necesidades para alcanzar un crecimiento rentable (Kim \& Mauborgne, 2005).

De acuerdo a Hoonsopon \& Ruenrom (2009), la innovación puede ser descrita en tres dimensiones: tecnología, consumidores y una tercera dimensión que podría tener elementos de las dos dimensiones de tecnología y consumidor. En la dimensión tecnológica, la innovación debe evaluarse con dos criterios: que sea nueva o diferente 
a tecnologías existentes, y única, es decir diferente a tecnologías actuales (Kristina \& Dean, 2005).

Desde la dimensión del consumidor, innovación es el grado en el que los productos son nuevos para el mercado objetivo y crean una oferta de beneficios para el consumidor (Ziamou \& Ratneshwar, 2003) así como soluciones para los problemas del mismo (Goldenberg, Lehmann \& Mazursky, 2001). Hoonsopon \& Ruenrom (2009) relacionan la innovación con el desarrollo de nuevos productos y demuestran que la innovación es un factor importante en el desempeño de los negocios, incrementando sus ventas, ganancias y ventajas competitivas

Por su parte, Miller (2006) genera un modelo que denomina $4 \mathrm{G}$ y que es muy similar al NexGen Systems que desarrolla Cooper. Este modelo implica que la innovación radical va más allá de las cuatro $P$ 's (producto [incremental], precio, plaza y promoción), y que para alcanzarla se deben añadir otras cuatro P's: producto (radical), procesos, gente y política. El autor habla de un proceso de innovación en espiral, y que define la gerencia de innovación como la capacidad de reunir gente, tecnología y procesos para crear valor a los consumidores, incluyendo una estructura organizacional nueva.

Finalmente, es importante mencionar lo que Osorio (2010) denomina el arte de fracasar para innovar con éxito. El autor desarrolla un modelo para innovar mediante aprendizaje por observación y mediante fallas generada por ciclos de prototipos y pruebas de mercado, y explica que estos nuevos procesos tendrán un efecto directo en la cultura, organización y gestión de recursos humanos en la empresa, preparándola para un cambio gradual en su ADN (ver Gráfico 3). Este autor percibe el modelo

Gráfico 3. Fases y etapas del proceso de innovación

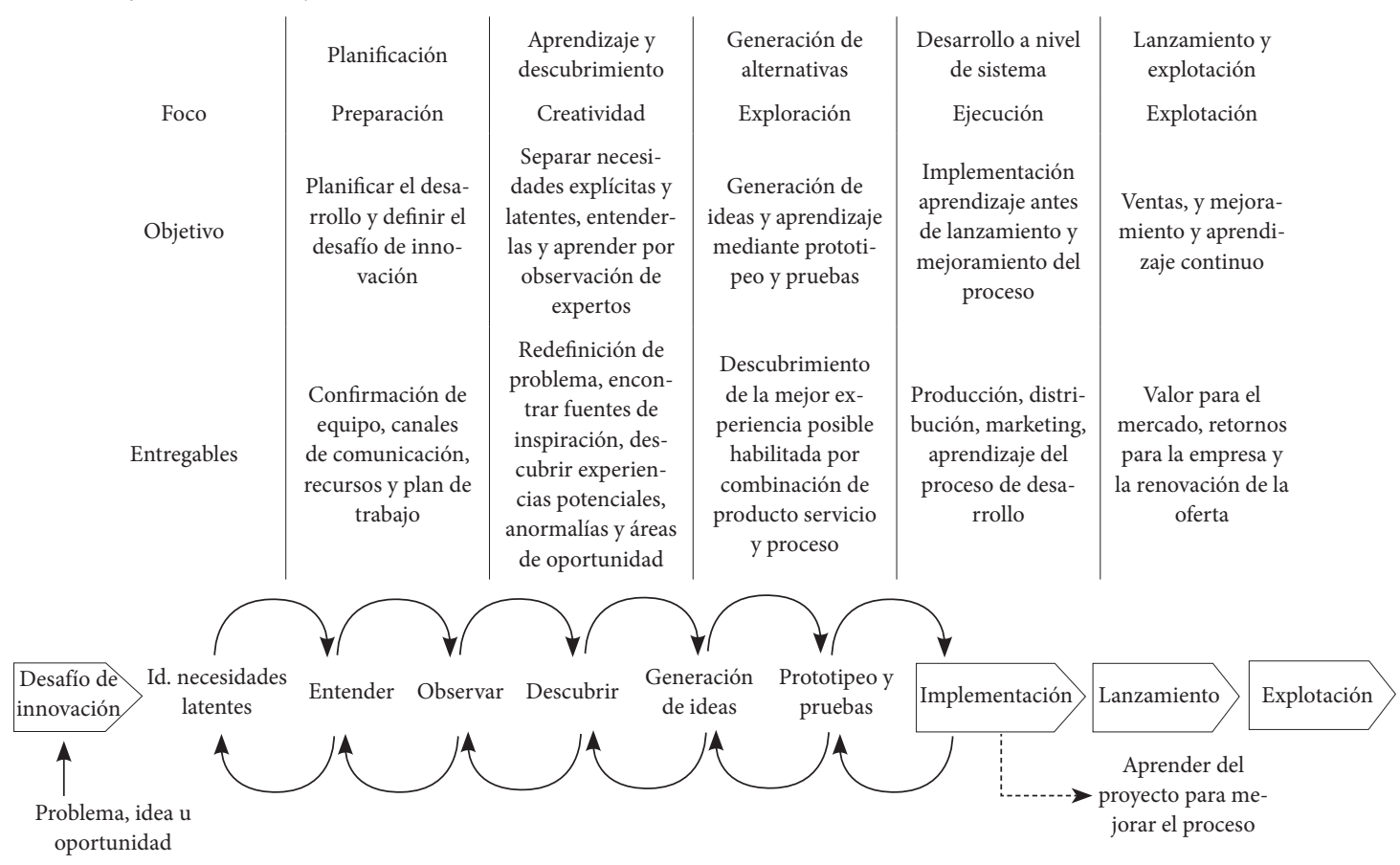

Fuente: De "El arte de fallar", de C.A. Osorio, 2010, Harvard Business Review, 88(4), p. 20. 
Stage-Gate como útil, pero algo rígido para la naturaleza caótica e iterativa propia de la innovación, y presenta su modelo como una alternativa. Su método es un proceso estructurado y orientado a focalizar el caos creativo en torno a un desafío de innovación, en producir mayores tasas de innovaciones disruptivas y en asegurar una mejor aceptación del mercado de los productos finales. El proceso es altamente iterativo, y permite que haya revisiones y saltos entre cualquiera de sus etapas.

A continuación se presentan los ocho rasgos que el autor desarrolla para ser incorporados al ADN de la empresa y mejorar la eficiencia en la innovación:

1 Sea como Da Vinci o Garfield, ambidiestro, es decir, la empresa debe generar ingresos mediante negocios existentes y al mismo tiempo mediante exploración de nuevos negocios;

2 Piense en desafíos, no en ideas, se deben generar portafolios de desafíos o problemas, luego se desarrollarán las ideas para resolverlos;

3 Planifique la innovación, una ejecución adecuada sin planificación es un fracaso;

4 Diseñe un calendario de desarrollo sin fechas, sino hitos, es demasiado rígido considerar un calendario fijo, es mejor considerar márgenes de tiempo;

5 No escuche al cliente, obsérvelo y viva con él, las investigaciones tradicionales limitan la innovación, es mejor acercarse;

6 Fallar no es permitido, es obligatorio, no hay mejor manera de innovar que fallar lo más temprano posible;

7 Invierta en fallar, como un seguro para no fracasar, dedicar un presupuesto para experimentar y desarrollar prototipos;

8 El cambio cultural no es el comienzo, es el resultado, el cambio cultural se da al adoptar nuevos procesos, el cambio resulta de la innovación y alimenta el nuevo proceso.
Es interesante como Osorio (2010) sostiene que el modelo de Stage-Gate no impulsa la innovación radical y presenta su modelo como una vía para incorporarla en el desarrollo de nuevos productos o, a través de fracasos iniciales (amplificando la ambigüedad, incertidumbre y riesgo asociados con el proyecto) antes de realizar las grandes inversiones. El autor explica que si se invierte luego de haber aprendido y haber reducido gran parte de los riesgos, se logran altos niveles de innovación.

Esto permite concluir que fracasar en las etapas iniciales del desarrollo de nuevos productos debe no sólo ser esperado, sino incentivado en las organizaciones. La fórmula de éxito es un balance entre rigurosidad y libertad, una combinación de una metodología paso a paso y rigurosa y, en las etapas tempranas del desarrollo de nuevos productos, un caos creativo centrado en un reto de innovación que lleve a fracasos tempranos, permitirá altos niveles de innovación y éxitos posteriores en el lanzamiento de los nuevos productos.

Es por ello que esta investigación explorará, a través de entrevistas en profundidad a una muestra de ejecutivos responsables en los procesos de desarrollo de nuevos productos, cómo es este proceso en las empresas colombianas seleccionadas y si existe este balance entre rigurosidad del método y caos para la innovación en las etapas tempranas; en caso contrario, se explorarán los obstáculos que impiden que estos ejecutivos implementen las mejores prácticas. Asimismo, se explorará el rol y el grado de impacto de la innovación en estos procesos desde la percepción de estos ejecutivos. Ambos factores permitirían extrapolar por qué continúan los fracasos en el desarrollo de nuevos productos; es decir, se evaluaría la variable latente, razones de fracaso a través de dos variables observables (a través de las 
entrevistas a los ejecutivos): implementación de metodologías en el desarrollo de nuevos productos y niveles de innovación el desarrollo de nuevos productos.

\section{Metodología}

La metodología utilizada para desarrollar el estudio fue de tipo cualitativo, la más recomendada dado el foco de interés de acuerdo con Lincoln \& Guba (1985). Estos autores sugieren que el estudio cualitativo es el más apropiado para comprender un tema desde la perspectiva de los participantes; en este caso, los participantes son os ejecutivos y gerentes responsables del proceso de desarrollo de nuevos productos en sus organizaciones. Asimismo, de acuerdo a Ambert, Adler, Adler \& Detzner (1995), la investigación cualitativa es más adecuada que la cuantitativa cuando la finalidad es descubrir y no verificar, adquirir información y aprender del comportamiento de la gente; $y$, dado que el objetivo de esta investigación es descubrir los obstáculos que enfrentan los ejecutivos en el proceso de desarrollo de nuevos productos, parece más apropiado el análisis cualitativo.

Específicamente se utilizaron entrevistas en profundidad, que son una forma no estructurada y directa de obtener información. Esta técnica de investigación es la más apropiada dada las características de la población en estudio y los objetivos a conseguir, pues la misma es recomendada en los casos de sondeo detallado del participante, de discusión de temas confidenciales, de entrevistas con profesionales y de entrevistas con competidores (Malhotra, Davila \& Treviño, 2004).

\subsection{Diseño de la investigación}

Para la realización de la entrevista se desarrolló una guía construida sobre el marco teórico que pretendía conocer los pasos seguidos por los ejecutivos para el lanzamiento de nuevos productos, las variables que recono- cen cómo ausentes y las variables que marcan el éxito y el fracaso de dichos lanzamientos. Para obtener dicha información, se les pidió a los entrevistados que documentaran casos específicos de experiencias de lanzamientos de nuevos productos, detallando los pasos o fases que la compañía tiene formalmente establecidos para el desarrollo de nuevos productos. Asimismo, se incluyeron preguntas para indagar sobre la percepción de la innovación y el rol de la misma dentro de dicho proceso.

Las entrevistas se realizaron en el segundo trimestre del año 2011 en la ciudad de Cali (Colombia). Las mismas fueron realizadas por estudiantes de décimo grado de la carrera de Mercadeo Internacional y Publicidad de la Universidad Icesi Los ejecutivos fueron entrevistados directamente en sus puestos de trabajo y las reuniones tuvieron una duración de aproximadamente una hora y media. Las entrevistas fueron grabadas y luego transcriptas de forma textual para su análisis e interpretación posterior.

La información recopilada fue analizada a través de análisis de contenido, metodología que puede ser utilizada para investigación cualitativa y cuantitativa (Collis \& Hussey, 2003; Harwood \& Garry, 2003; Neuendorf, 2002). Cuando se utiliza para análisis de estudios cualitativos, usualmente ocurre en etapas tempranas de la investigación o para el desarrollo de futuras hipótesis (Harwood \& Garry, 2003), que es justamente el foco de esta investigación. Las descripciones realizadas por los participantes se transcribieron textualmente y se codificaron siguiendo el procedimiento para el análisis de contenido cualitativo sugerido por Strauss \& Corbin (1990).

Posteriormente, se inició la labor de análisis con la selección de las unidades de información o los fragmentos relevantes. Seguidamente, se organizó, agrupó y reagru- 
paron los fragmentos seleccionados, procedimiento denominado por Strauss \& Corbin (1990) como codificación axial. Por último, se establecieron e identificaron las categorías de análisis y los fragmentos que las conformaban y se procedió a la interpretación del contenido de los mismos.

\subsection{Definición de la muestra}

La conformación de la muestra se realizó cumpliendo los criterios de exhaustividad (Mariampolski, 2001), tomando a empresas de diferentes industrias y líderes dentro de su sector. Asimismo, los ejecutivos seleccionados debían tener amplia experiencia en el área de mercadeo, específicamente más de cinco años de experiencia en desarrollo de nuevos productos y ser empleados de medio a alto rango.

La estrategia para la selección de los participantes fue teórica (Taylor \& Bogdan, 1986) o intencional (Coffey \& Atkinson, 2003; Lincoln \& Guba, 1985); es decir, la escogencia de los participantes se estableció a partir de los propósitos de la investigación. Así se eligieron doce gerentes y ejecutivos, los cuales trabajaban en organizaciones pertenecientes a diferentes industrias: aseo del hogar, alimentos, dulces, farmacéuticos, muebles e industria azucarera. Dichas empresas son organizaciones grandes, nacionales y multinacionales, algunas con casa matriz en Colombia, como en los casos de Colgate Palmolive, Reckitt Benckiser, Cadbury Adams, Rica Rondo, Grupo Nutresa, BSN Medical, Casa Oben, Carvajal e Incauca.

\section{Resultados}

\subsection{Causas de fracaso}

Del análisis de las doce entrevistas se dedujeron las siguientes variables como factores que afectan el buen desempeño del desarrollo de nuevos productos en el caso colombiano: a. Nuevos productos, la panacea del crecimiento. No se dedica el tiempo necesario a cada fase del proceso de desarrollo de nuevos productos por la presión ejercida en la organización para obtener resultados inmediatos, lo que hace que la toma de decisiones se realice sin el soporte ni el análisis de información adecuado.

b. Bajo nivel de innovación en los nuevos productos. La mayoría de las propuestas no se diferencian de los productos existentes, es decir, pocos productos son percibidos como realmente innovadores por el mercado.

c. Poca orientación al consumidor. En muchos casos hay debilidades en las investigaciones de mercado, en términos de enfoque, interpretación y aplicación, lo que lleva a la empresa a tomar decisiones equivocadas. Como mencionan algunos del os entrevistados, es necesario aprender y desaprender cosas que están muy arraigadas, lo cual es especialmente importante para empresas multinacionales a las que se les dificulta la adaptación de sus marcas internacionales al mercado local. Esto puede traer como resultado que el nuevo producto no llene las expectativas del consumidor.

d. Falta de trabajo en equipo y cultura de innovación. La mayoría de los entrevistados mencionan la falta de coordinación, sinergia, comunicación y trabajo en equipo de las diferentes áreas lo que lleva a ignorar variables importantes durante todo el desarrollo de nuevos productos.

e. Debilidades en la identificación y medición de nuevas oportunidades de mercado. Generalmente las ideas exploradas se desarrollan sobre la base de los productos y las categorías existentes, olvidando que los mercados no necesariamente son océanos en calma. Este factor está relacionado con debilidades en el planteamiento estratégico de la investigación de mercados. 
f. Las compuertas no se cierran. Los criterios seleccionados para pasar de una fase a otra son débiles y adicionalmente no hay una figura de gerencia sénior comprometida en hacerlos cumplir. Las ideas fluyen fácilmente desde la generación hasta el lanzamiento sin que se detecten deficiencias claves del proyecto y se detengan a tiempo.

g. No se realiza confirmación del pronóstico de demanda. Las organizaciones no utilizan la herramienta de la prueba piloto para ajustar las proyecciones de venta ni para retroalimentar el proceso de desarrollo de nuevos productos, incluyendo los correctivos en la propuesta de valor.

h. Estrategia de mercadeo que no soporta el lanzamiento del nuevo producto. Existen debilidades en la formulación del plan, implementación y seguimiento del mismo para la aplicación de correctivos. Esto trae como consecuencia debilidad en construcción de posicionamiento, estrategias inadecuadas de precio y canal, lo que hace que el consumidor no perciba la innovación en el producto. i. Inversión insuficiente de recursos en la fase de lanzamiento. Luego de haber realizado una inversión importante en la generación de la idea, el prototipo y el lanzamiento del nuevo producto, la compañía no destina lo suficiente para que el producto pase de interrogante a estrella, lo que no permite el tiempo para enseñar, convencer, cambiar hábitos en el consumidor y construir la marca.

Esta lista de variables es similar a la recopilada en la sección de soporte teórico de este trabajo, lo que permitiría pensar que el fenómeno de fracaso de nuevos productos es universal. Colombia no parece presentar diferencias relevantes respecto a los casos estudiados por los autores mencionados. Sin embargo, es importante resaltar que cada uno de los ejecutivos entrevistados reportaba sólo entre tres y cuatro variables, lo que refleja que los mismos no tienen una visión holística de la situación, lo que explicaría en parte las tasas de fracaso en el desarrollo de nuevos productos. Adicionalmente, el aprendizaje de desarrollo de nuevos productos se lleva a cabo a través de su experiencia y no necesariamente están expuestos a modelos conceptuales como los presentados en este estudio; de hecho, sólo un entrevistado conoce y ha implementado el modelo Stage-Gate en la organización en la que trabaja.

En cuanto a los factores de éxito, se observó que los entrevistados generalmente hacían referencia a que subsanando los factores de fracaso, se alcanzaba el éxito; es decir, que la lista de factores de éxito coincidiría con desarrollar satisfactoriamente cada uno de las variables mencionada como causante de fracaso.

\subsection{Metodología de desarrollo de nuevos productos}

En cuanto al proceso de lanzamiento que los entrevistados exponen, se puede afirmar que en general los procesos desarrollados en las empresas carecen de varias fases importantes del desarrollo de nuevos productos, presentando procesos incompletos. Sólo dos empresas tienes procesos formales que contemplan las fases típicas de un modelo Stage-Gate. Específicamente, la fase que con mayor frecuencia se omite es la revisión de seguimiento post-lanzamiento y se evidencian fuertes debilidades en la fase cuatro de prueba y validación de producto, la cual incluye un análisis profundo de factibilidad comercial, técnica y económica del producto.

Estos resultados permitirían inferir que hay un desconocimiento del estado del arte en desarrollo de nuevos productos por parte de estos ejecutivos, los que en su mayoría han desarrollado un proceso intuitivo pero con desconocimiento de las últimas metodologías diseñadas para reducir la 
tasa de fracaso, por ejemplo, desconocen el modelo Stage-Gate o la importancia de las compuertas, que abren o cierran el flujo de avance de los proyectos. También se extrapola que el aprendizaje es producto de las experiencias de los ejecutivos, pero que este conocimiento no necesariamente se traslada a la empresa y cuando el ejecutivo se va, el aprendizaje se va con él, es decir, las organizaciones no aprenden (Senge, 1990).

\subsection{Percepción de innovación y aplicación al desarrollo de nuevos productos}

Para los entrevistados, innovación es algo nuevo, diferente a lo existente en el mercado y que agrega valor al consumidor, como mencionó uno de los entrevistados: "Si un invento no logra suplir una necesidad de la vida cotidiana deja de ser una innovación, simplemente es un invento, hay miles de inventos." ${ }^{4} \mathrm{La}$ innovación se percibe como una herramienta enfocada al área comercial, siendo su aplicación principal el mejoramiento y lanzamiento de nuevos productos: "se supone que en mercadeo se da el ambiente propicio para la creación de conceptos nuevos y de allí surgen las ideas asociadas con producto."5 Sólo uno de los entrevistados percibe la innovación como una estrategia que puede abarcar todas las áreas de la empresa.

En cuanto al grado de innovación en las empresas, los ejecutivos perciben que en sus organizaciones se desarrollan innovaciones de grado 3 hasta 5 , en una escala del 1 al 5 , donde 1 son innovaciones incrementales y 5 innovaciones radicales. Sin embargo, se evidencia un desconocimiento del tema y de los diferentes grados de innovación, por ejemplo, uno de los entrevistados menciona: "la calificaría en una alto grado, porque la empresa investiga muy bien al consumidor

4 Cita textual a uno de los entrevistados del estudio.

5 Cita textual a uno de los entrevistados del estudio.
6 Cita textual a uno de los entrevistados del estudio.

7 Cita textual a uno de los entrevistados del estudio.

8 Cita textual a uno de los entrevistados del estudio. y puede encontrar oportunidades de mejoramiento de las categorías donde compite y puede generar esa innovación a través de la diferenciación." Adicionalmente, en el reporte de casos documentados de innovación, se ratifica que la mayoría de estos podrían clasificarse en el rango de innovación incremental y no de innovación radical.

Cuando se les pregunta a los ejecutivos sobre el rol e impacto de la innovación en el desarrollo de nuevos productos, todos coinciden en señalar que no debe existir desarrollo de nuevos productos sin innovación. Asimismo, conciben la innovación como una variable que impacta las ventas y que, en el largo plazo, permite maximizar los recursos, por ejemplo: "es esencial; toda la inversión en tiempo, esfuerzo humano y dinero no tiene sentido si se hace sobre algo que ya existe, el impacto en el consumidor será tangencial y realmente no creará la energía que requiere un producto para ser exitoso durante una etapa de lanzamiento." Por supuesto, todas estas implicaciones se conciben dentro de su "espacio perceptual de innovación" "que está cerca de las fronteras de innovaciones incrementales.

Finalmente, cuando se les pregunta a los entrevistados sobre los factores que afectan o bloquean la innovación en sus empresas, mencionan consistentemente debilidades en la cultura de innovación; específicamente, mencionan que no se dedican recursos (económicos, humanos y tiempo), no hay un compromiso de la alta gerencia, hay alta resistencia al cambio, normas rígidas y burocracia. En segundo lugar, se mencionan fallas en el diagnóstico de las necesidades de los clientes, y por último, la aversión al riesgo o el costo en el que deben incurrir para desarrollar un nuevo producto, es decir,

ESTUDIOS GERENCIALES VOL. 28 EDICIÓN ESPECIAL 
el temor al fracaso. A continuación algunos verbatims ${ }^{9}$ de los ejecutivos entrevistados que apoyan estos hallazgos:

- "es cuestión de actitud de la alta gerencia, cuando los gerentes generales, la gente de primer nivel de gestión son timoratos, o sea hay mucha aversión al riesgo, la innovación no funciona y esas compañías están condenadas, compañías muy conservadoras estas condenadas"

- "a veces, la resistencia al cambio que tienen ciertas personas y las limitaciones de presupuesto para desarrollar nuevas ideas y la resistencia al cambio de la gente, básicamente esas dos cosas"

- "según mi experiencia los bloqueadores más fuertes a la innovación son: los procedimientos rígidos, los empleados de mente cerrada, las compañías que toman decisiones únicamente basados en cifras positivas de evaluaciones controladas, la falta de empoderamiento y los ambientes laborales hostiles"

- "que los líderes que tiene son convergentes, entonces por qué no hay innovación, porque

9 Citas textuales tomadas de los entrevistados del estudio. no es que no hagan nada, si hacen, sino que hacen lo mismo que hace todo el mundo y al final del día la única diferenciación que tiene y que pueden esgrimir es precio"

Para consolidar este análisis e integrar los hallazgos con el soporte teórico recopilado, se elaboró la Tabla 2, en ella se resumen los factores claves a gerenciar para un desarrollo de nuevos productos de acuerdo a las mejores prácticas. $\mathrm{Al}$ aplicar estos factores a las empresas estudiadas y haciendo una evaluación con una escala del 1 al 5 (donde 1 es deficiente y 5 excelente), los resultados obtenidos son bastante bajos. Esto indica que las empresas colombianas tienen muchas áreas de oportunidad en lo que el desarrollo de nuevos productos se refiere.

Asimismo, la Tabla 2 puede ser utilizada como una herramienta práctica para que las empresas auditen sus procesos de desarrollo de nuevos productos y, con base en los resultados, puedan desarrollar planes para mejorar el proceso de desarrollo de nuevos productos, empezando por aquellos factores donde su evaluación sea deficiente.

Tabla 2. Factores clave en el desarrollo de nuevos productos: nivel de las mejores prácticas en las empresas colombianas

\begin{tabular}{l} 
Factor clave DNP \\
\hline Recursos económicos \\
Recursos humanos (dedicados) \\
Estrategia corporativa (compromiso alta gerencia) \\
Estrategia de mercadeo (apoyo lanzamiento) \\
Trabajo en equipo (coordinación de todos los departamentos) \\
Implementación impecable \\
Procesos de DNP (pasos-compuertas) \\
Medición (durante el proceso y de resultados) \\
Nivel de innovación (caos creativo - fallas como aprendizaje) \\
Orientación al cliente/mercado (identificación de oportunidades)
\end{tabular}

Fuente: Elaboración propia. 


\section{Conclusiones}

Dada las limitaciones metodológicas de este estudio (carácter exploratorio y muestra no representativa), esta investigación permite tener una primera aproximación de cómo se llevan a cabo los procesos de desarrollo de nuevos productos en Colombia y el rol de la innovación en los mismos. Las siguientes conclusiones son hipótesis sólidas que servirán como base para un futuro estudio confirmatorio.

En primer lugar, no se encontró evidencia de nuevas recetas que se apliquen en Colombia, diferentes a las metodologías ya desarrolladas en la literatura de desarrollo de nuevos productos. En todo caso, las mismas son precarias, limitadas e incompletas, lo que evidencia un vacio conceptual de know how en los ejecutivos en relación a muchas de estas metodologías, como es el caso del NexGen State-Gate. En ese caso, los procesos de desarrollo de nuevos productos son intuitivos y están muy afectados por la presión de tiempo y recursos de las organizaciones, lo cual lleva a tomar decisiones a lo largo del proceso sin el suficiente soporte. Este hallazgo sugiere que las empresas en Colombia deberían invertir en capacitación y actualización de sus ejecutivos en estos temas, porque aunque la experiencia es muy importante, en la academia se desarrollan métodos y conceptos que pueden fortalecer y subsanar las debilidades en el desarrollo de nuevos productos.

En segundo lugar, dado que la innovación es un elemento esencial en el éxito del desarrollo de nuevos productos, se explica que muchos de los fracasos de estos desarrollos están relacionados con la visión y práctica limitada de la misma, la cual se centra en la división comercial y en el rango de innovación incremental. Es por ello que las empresas deberían concientizar que la innovación no es la panacea de la empresa que llega a solucionar sus problemas, sino que más bien, es un proceso de aprendizaje que conlleva a un cambio a nivel de cultura organizacional donde la innovación es un proceso sinérgico, con involucración de todas las áreas y holístico con apoyo de la alta gerencia, a través de su compromiso y la asignación de los recursos adecuados. Mientras esto no suceda, los ejecutivos seguirán desgastándose, tratando de lograr diferenciaciones centradas en mejoras incrementales que sólo permanecen en el corto plazo, lo que los vuelve muy vulnerables ante la compleja dinámica de los mercados.

En tercer lugar, y tal vez la conclusión más importante, está relacionada con la pregunta ¿Qué hace que los ejecutivos se sigan equivocando? Podría considerarse que parte de esta pregunta puede responderse con las debilidades mencionadas en las dos primeras conclusiones elaboradas, pero definitivamente hay otros elementos claves que están relacionados entre sí. Las empresas no están logrando construir un aprendizaje sobre sus fracasos, más aún la cultura organizacional está enfocada al éxito, lo que los hace ser adversos a ensayar o descubrir nuevas fronteras. Las fallas son vistas como fracasos y no como oportunidades de aprendizaje, por lo que no se toman el tiempo de reflexionar, compartir y apropiarse de las lecciones para incrustarlas en el ADN de las empresas.

Esta cultura, enfocada al éxito con exclusión del error, nubla la visión de entender que hay resultados que requieren tiempo e inversión dificultando que la empresa emprenda proyectos de innovación radicales. Asimismo, la intolerancia al fracaso y la urgencia de resultados inmediatos bloquea el potencial creativo de sus empleados e incluso no les permite recordar que la teoría funciona, es decir, en muchas ocasiones 
aunque conozcan la esencia de los conceptos y lo que en teoría debería hacerse, terminan tomando decisiones diferentes por presiones de tiempo y recursos.

En este sentido, es muy importante para las empresas entender que no podrán ser innovadoras y lograr éxito en el desarrollo de nuevos productos si no cambian su percepción y abrazan el fracaso, ya no como desaciertos vergonzosos, sino como fuente de aprendizaje. Las organizaciones podrían planificar reuniones sistemáticas para revisar el inventario de sus errores y las lecciones aprendidas para retroalimentar sus procesos de desarrollo de nuevos productos y lograr mayores tasas de éxito. La receta del éxito en el desarrollo de nuevos productos debería contener un balance entre adoptar metodologías y procesos que permitan desarrollar todas las actividades importantes y evaluarlas constantemente para detener el flujo en caso de que sea necesario; pero al mismo tiempo, deben ser lo suficientemente flexibles para permitir enfrentar retos más que generar ideas y regresar sobre errores cuantas veces sea necesario. Ese balance no es el mismo para todas las empresas, cada uno deberá encontrar su propio balance dependiendo de su cultura, de su estructura $y$ del mercado en el que compite.

\section{Referencias bibliográficas}

Ambert, A., Adler, P.A., Adler, P. \& Detzner, D. (1995). Understanding and Evaluating Qualitative Research. Journal of Marriage \& Family, 57(4), 879-893.

Booz, Allen, \& Hamilton. (1982). New Product Management for the 1980s. New York, NY: Autores.
Brody, A. (1999). Developing New Food Products for a Changing Marketplace. Duluth, MI: CRC Press.

Chandy, R. \& Tellis, G.J. (2000). The incumbent's curse? Incumbency, size, and radical product innovation. Research Technology Management, 64(3), 1-17.

Clancy, K. \& Shulman, S. (1994). Mitos del marketing que están matando a los negocios. México: McGraw-Hill.

Coffey, A. \& Atkinson, P. (2003). Encontrar sentido a los datos cualitativos. Estrategias complementarias de investigación. Medellín, Colombia: Universidad de Antioquia.

Collis, J. \& Hussey, R. (2003). Business research ( $2 \mathrm{a}$ ed.). Basingstoke, NH: Palgrave Macmillan.

Cooper, R. (1990). Stage-Gate Systems: A New Tool for managing new Products. Business Horizons, 33(3), 44-54.

Cooper, R. (2006). Formula for Success. Marketing Management Magazine, March/ April, 21-24.

Cooper, R. (2007). Winning business in product development: the critical success factor. Research Technology Management, $50(3), 52-66$.

Cooper, R. (2008). Perspective: The StageGate ${ }^{\circ}$ Idea-to Launch Process-Update, What's New, and NexGen Systems. Journal of Product Innovation Management, 25(3), 213-232.

Cooper, R. (2009). Effective Gating. Make productive by using gates with teeth. Marketing Management Magazine, March/ April, 12-17.

Cooper, R. \& Kleinschmidt, E. (1993). StageGate Systems for New Product Success. Marketing Management Magazine, 1(4), 20-29.

Crawford, M. \& Di Benedetto, A. (2003). New Products Management. New York, NY: McGraw-Hill. 
Edgett, S. (2007). Portfolio Management: Optimizing for Success. Houston, TX: American Productivity \& Quality Center. Goldenberg, J., Horowitz, R., Levav, A. \& Mazursky, D. (2003). Finding your innovation sweet spot. Harvard Business Review, 81(3), 3-10.

Goldenberg, J., Lehmann, D. \& Mazursky, D. (2001). The Idea itself and the Circumstances of its Emergence as Predictors of New product Success. Management Science, $47(1), 69-84$.

Harwood, T. \& Garry, T. (2003). An Overview of Content Analysis. The Marketing Review, 3(4), 479-498.

Hoonsopon, D. \& Ruenrom, G. (2009). The Empirical Study of the Impact of Product Innovation Factors on the Performance of New Products: Radical and Incremental Product Innovation. The Business Review, 12(2), 155-161.

Kim, W.C. \& Mauborgne, R.A. (2005). Blue Ocean Strategy from theory to practice. California Management Review, 47(3), 105-121.

Kristina, B. \& Dean, M. (2005). When is an Invention Really radical? Defining and Measuring Technological Radicalness. Research Policy, 34(5), 717-737.

Kuczmarski, T. (1997). Innovación: estrategias de liderazgo para mercados de alta competencia. Bogotá: McGraw-Hill.

Lincoln, Y. \& Guba, E. (1985). Naturalistic inquiry. Beverly Hills, CA: Sage.

Malhotra, N., Davila, J.F. \& Treviño, M. (2004). Investigación de Mercados. Un enfoque aplicado. Madrid: Pearson Education.

Mariampolski, H. (2001). Qualitative Market Research. Londres: Sage.

Miller, W. (2006). Innovation Rules! Research Technology Management, 49(2), 8-14.
Neuendorf, K.A. (2002). The content analysis guidebook. Londres: Sage.

Osorio, C.A. (2010). El arte de fallar. Harvard Business Review, 88(4), 76-85.

Ries, A \& Trout, J. (1989). La revolución del marketing: La táctica dicta la estrategia. Bogotá: McGraw-Hill.

Schnarch, A. (2009). Desarrollo de nuevos productos y empresas. Bogotá: McGrawHill.

Schumpeter, J. (1978). Teoría del desenvolvimiento económico ( $\left.5^{\mathrm{a}} \mathrm{ed}\right)$. México. Fondo de Cultura económica.

Senge, P. (1990). The Fifth Discipline: The Art and Practice of the Learning Organization. New York, NY: Doubleday \& Company.

Sethi, R., Smith, D.C. \& Park, C.W. (2001). Cross-Functional Product Development Teams, creativity, and The Innovativeness of New Consumer Products. Journal of Marketing Research, 38(1), 73-85.

Strauss, A. \& Corbin, J. (1990). Basic of qualitative research. Newbury Park, CA: Sage. Taylor, J. (1983). Como planear estrategias rentables de productos nuevos. New York, NY: Modern Business Reports.

Taylor, S. \& Bogdan, R. (1986). Introducción a los métodos cualitativos de investigación: La búsqueda de significados. Buenos Aires: Paidós.

Urban, G.L. \& Hauser, J.R. (1980). Design and marketing of new products. New Jersey, NJ: Prentice-Hall.

Ziamou, P. \& Ratneshwar, S. (2003). Innovations in Products Functionality: When and Why Are Explicit Comparisons effective? Journal of Marketing, 67(abril), 49-61. 